\title{
Comparison of cochlear delay estimates using otoacoustic emissions and auditory brainstem responses
}

Harte, James; Pigasse, Gilles; Dau, Torsten

Published in:

Acoustical Society of America. Journal

Link to article, DOI:

$10.1121 / 1.3168508$

Publication date:

2009

Document Version

Publisher's PDF, also known as Version of record

Link back to DTU Orbit

Citation (APA):

Harte, J., Pigasse, G., \& Dau, T. (2009). Comparison of cochlear delay estimates using otoacoustic emissions and auditory brainstem responses. Acoustical Society of America. Journal, 126, 1291-1301.

https://doi.org/10.1121/1.3168508

\section{General rights}

Copyright and moral rights for the publications made accessible in the public portal are retained by the authors and/or other copyright owners and it is a condition of accessing publications that users recognise and abide by the legal requirements associated with these rights.

- Users may download and print one copy of any publication from the public portal for the purpose of private study or research.

- You may not further distribute the material or use it for any profit-making activity or commercial gain

- You may freely distribute the URL identifying the publication in the public portal 


\title{
Comparison of cochlear delay estimates using otoacoustic emissions and auditory brainstem responses
}

\author{
James M. Harte, ${ }^{\text {a) }}$ Gilles Pigasse, and Torsten Dau \\ Department of Electrical Engineering, Centre for Applied Hearing Research, Technical University of \\ Denmark, 2800 Kongens Lyngby, Denmark
}

(Received 21 November 2008; revised 10 June 2009; accepted 14 June 2009)

\begin{abstract}
Different attempts have been made to directly measure frequency specific basilar membrane (BM) delays in animals, e.g., laser velocimetry of BM vibrations and auditory nerve fiber recordings. The present study uses otoacoustic emissions (OAEs) and auditory brainstem responses (ABRs) to estimate BM delay non-invasively in normal-hearing humans. Tone bursts at nine frequencies from 0.5 to $8 \mathrm{kHz}$ served as stimuli, with care taken to quantify possible bias due to the use of tone bursts with different rise times. BM delays are estimated from the ABR latency estimates by subtracting the neural and synaptic delays. This allows a comparison between individual OAE and BM delays over a large frequency range in the same subjects, and offers support to the theory that OAEs are reflected from a tonotopic place and carried back to the cochlear base via a reverse traveling wave. (C) 2009 Acoustical Society of America. [DOI: 10.1121/1.3168508]
\end{abstract}

PACS number(s): 43.64.Jb, 43.64.Ri, 43.64.Kc [BLM]

Pages: 1291-1301

\section{INTRODUCTION}

The human auditory system is known to possess high frequency selectivity and to compress a wide range of sound levels into an audible range. These characteristics can be explained by the mechanical properties of the inner ear. The tonotopic organization of the cochlea is such that highest frequencies are processed at the base of the basilar membrane (BM) and the lowest frequencies are processed at the apex. This is due to a stiffness gradient along the BM. The inward traveling wave has a longer path to travel to reach the low-frequency region of the cochlea compared to the highfrequency region (von Békésy, 1960). There is an intrinsic relation between frequency and travel time in the cochlea, defined as the cochlear delay, $\tau_{\mathrm{BM}}(f)$. Different attempts have been made to directly measure BM vibration, e.g., by measuring the reflections of a laser Doppler velocimeter on the BM of animals and human cadavers (e.g., Recio et al., 1998; Goodman et al., 2004). Opening the cochlea, however, decreases its sensitivity at low frequencies (Dong and Cooper, 2006), and this only provides an approximation of the normally functioning human cochlea. Various measurement techniques have been developed to non-invasively and indirectly record BM delay such as otoacoustic emissions (OAEs) (Norton and Neely, 1987; Şerbetçioğlu and Parker, 1999) and auditory brainstem responses (ABRs); (Gorga et al., 1988; Murray et al., 1998). Comparing cochlear delay estimated from both these measures, in the same subject group, can provide valuable knowledge about the physical generation mechanisms of both. Such experimental data are, so far, limited in normal-hearing listeners, only extending across the mid-frequency region of the cochlea.

\footnotetext{
a) Author to whom correspondence should be addressed. Electronic mail: jha@elektro.dtu.dk
}

\section{A. OAEs}

OAEs are low-level signals originating in the cochlea, traveling through the middle ear and recorded in the ear canal (EC). This epiphenomenon of a normally functioning auditory system was first recorded in humans by Kemp (1978). Classically, OAEs have been classified in terms of the stimulus with which they are evoked, i.e., tones, tonal complexes, transients, or no stimulus at all. When transient signals like clicks or tone-bursts (TBs) are used, the recorded OAEs are called transient evoked OAEs (TEOAEs). Studies have also shown that OAEs have a spectrum similar to that of the evoking tone burst (Wit and Ritsma, 1980; Kemp et al., 1986). However, TEOAE spectra are often dominated by a few resonant peaks or dominant frequencies, often associated with measurable spontaneous OAEs (Jedrzejczak et al., 2008). If present, these may confound estimates of latency for the OAE recordings. Previous studies have investigated the latencies of TEOAEs in the time domain (Norton and Neely, 1987; Şerbetçioğlu and Parker, 1999; Kapadia and Lutman, 2000; Hoth and Weber, 2001; Goodman et al., 2004; Thornton et al., 2006) and in the time-frequency domain (Elberling et al., 1985; Probst et al., 1986; Tognola et al., 1997; Lucertini et al., 2002; Sisto and Moleti, 2002; Jedrzejczak et al., 2005). Unlike clicks which are broadband stimuli, TBs offer the advantage of being limited in frequency and can therefore be used to investigate limited regions of the cochlea. However, they are therefore less precisely defined in time making latency estimation more difficult. A number of studies have investigated TB evoked OAE (TBOAE) latencies in the time domain, using TBs over a limited frequency range (Wilson, 1980a; Grandori, 1985; Şerbetçioğlu and Parker, 1999; Norton and Neely, 1987).

Kalluri and Shera (2007) demonstrated the near equivalence of TEOAEs and stimulus frequency OAEs (SFOAEs), when the stimulus intensity is given in a bandwidthcompensated sound pressure level (SPL). The most accepted 
hypothesis for the generation of SFOAEs at low to moderate excitation levels is the coherent reflection filtering (CRF) theory (Zweig and Shera, 1995). The theory states that randomly distributed inhomogeneities in the BM local impedance reflect microscopic wavelets from the forward traveling wave (Shera and Guinan, 1999; Kalluri and Shera, 2007). These wavelets sum up in-phase around the peak of the traveling wave and form a so-called retrograde or backwards traveling wave.

\section{B. ABRs}

Cochlear activity and therefore the cochlear delay, $\tau_{\mathrm{BM}}$, is also reflected at stages higher than the cochlea in the human auditory pathway. Auditory evoked potentials can be used to obtain an indirect estimate of cochlear delay in humans (Neely et al., 1988). Specifically, ABRs are generated above the cochlea and are the result of the simultaneous activation of nerve cells in the brainstem (Møller, 1994). A typical ABR is made up of a series of so-called waves, whose latency is linked with the distance between the cochlea and the source of this wave. Waves I, III, and V are typically the most pronounced waves. The exact physiological sources of these waves are not fully known in humans. However, it is generally accepted that wave I stems from the distal portion of the afferent cochleo-vestibular nerve (VIIIth nerve), as demonstrated by Jewett and Williston (1971) and Møller and Jannetta (1983). It is assumed that wave III arises from an area near or inside the cochlear nucleus, situated at the bottom of the brainstem, and that wave V is attributed to neuronal activities between the lateral lemniscus and the inferior colliculus, contralateral to the side of the stimulus (Møller, 1994). Wave V is the wave with the largest amplitude and hence the most easily detectable. In order to obtain an estimate, $\hat{\tau}_{\mathrm{BM}}^{(\mathrm{ABR})}$, of the cochlear delay, all of the component latencies must be considered. Wave- $\mathrm{V}$ latency is often considered to be composed of the sum of the synaptic delay, $\tau_{\text {synaptic }}$, the neural delay, $\tau_{\text {neural }}$, as well as the cochlear delay $\tau_{\mathrm{BM}}$ (Neely et al., 1988). Thus, an estimate of the cochlear delay can be given by

$$
\hat{\tau}_{\mathrm{BM}}^{(\mathrm{ABR})}=\tau_{\text {wave } \mathrm{V}}-\tau_{\text {neural }}-\tau_{\text {synaptic }} .
$$

The synaptic delay is the time to be estimated between the inner hair-cells activity and the auditory-nerve fibers firing. It is typically around $1 \mathrm{~ms}$ (Kiang, 1975; Kim and Molnar, 1979; Møller and Jannetta, 1983; Burkard and Secor, 2002) and assumed to be independent of frequency and level (Don et al., 1998). The neural conduction time (neural delay) is the time between the auditory-nerve activity and the place generating the $\mathrm{ABR}$ wave. It can be estimated from the interpeak delays of the ABR, i.e., by the latency difference of wave I and wave V: $\tau_{\text {neural }}=\Delta_{\mathrm{I}-\mathrm{V}}$. However, unlike wave V, the detection of wave I is rather difficult due to its small amplitude. It was therefore decided in this study to detect wave III instead and use the assumption $\Delta_{\mathrm{I}-\mathrm{V}}=2 \Delta_{\mathrm{III}-\mathrm{V}}$ (Don and Eggermont, 1978; Eggermont and Don, 1980; Don and Kwong, 2002). The cochlear delay estimate, $\hat{\tau}_{\mathrm{BM}}^{(\mathrm{ABR})}$, can then be calculated for each subject, using individual estimates of the wave- $\mathrm{V}$ latency and the interpeak delay:

$$
\hat{\tau}_{\mathrm{BM}}^{(\mathrm{ABR})}=\tau_{\text {wave } \mathrm{V}}-2 \Delta_{\mathrm{III}-\mathrm{V}}-1 \mathrm{~ms} .
$$

When measuring cochlear delays, the propagation time in the outer- and middle-ear is typically neglected because it is very short and not frequency dependent, unlike the cochlear delay. This assumption will be revisited in Sec. IV.

Historical experimental studies have demonstrated that $\tau_{\mathrm{BM}}$ decreases as a power law of frequency. Neely et al. (1988) used the formula $\tau_{\mathrm{BM}} \propto b c^{-i} f^{-d}$ to describe the latency of $\mathrm{ABR}$ as a function of frequency normalized to $1 \mathrm{kHz}, f$ in $\mathrm{kHz}$, with $i$ representing the tone burst intensity in $\mathrm{dB}$ SPL divided by 100 , and $b=12.9 \mathrm{~ms}, c=5.0 \mathrm{~ms}$, and $d=0.413$ representing fitting constants.

\section{Goals and scope of the study}

The main objectives of this study were to estimate the cochlear delay over a broader frequency range than historically carried out, and to determine the most appropriate method to do so. OAEs and ABRs were measured for the same subjects, using the same stimuli in the same laboratory, in an attempt to fill the dearth of experimental data pointed out by Moleti and Sisto (2008). In the present study, TBs ranging from 0.5 to $8 \mathrm{kHz}$ were used and the implicit assumptions required to convert $\mathrm{ABR}$ latencies to cochlear delay are discussed. This study also compares OAE and ABR latency accumulation and tests the consistency with current theories about the OAE generation mechanisms. The CRF theory for OAE generation allows prediction upon the build up of delay in the final OAE recorded in the EC. At its simplest, the CRF theory suggests that the OAE delay is twice the delay between the stimulus onset and the peak of the (forward) traveling wave (Zweig and Shera, 1995). From this simple prediction, some debate has appeared in the literature to challenge the CRF theory, on the basis that the observed delay is too short. This will be discussed in the light of the experimental results from the present study. However, it should be noted that in this paper physical transmission delay is measured and not the relation between phase and frequency predicted by CRF theory. If the relation $\tau_{\mathrm{OAE}}=2 \tau_{\mathrm{BM}}$ is obtained, then this implies that the $\mathrm{OAE}$ is generated at the tonotopic place, and propagates backwards to the base as a reverse traveling wave, with the same speed as that of the forward path. If this relation is not found, then this would imply that either the backward transmission is faster, as recently suggested by Ren (2004), or the backward wave is generated at a more basal cochlear place.

\section{METHODS}

\section{A. Subjects}

The subjects participating in this experiment were 11 normal-hearing adults: 2 females, 9 males, aged between 22 and 30 years. The different numbers of male and female subjects might bias the result. However, the present study compares $\mathrm{OAE}$ and $\mathrm{ABR}$ latencies on an individual basis. The higher number of male subject might lead to longer latencies compared to other studies (Don et al., 1993). All subjects had pure-tone thresholds better than $15 \mathrm{~dB}$ HL in the range $0.25-8 \mathrm{kHz}$. 
TABLE I. TB stimuli used, with length in $\mathrm{ms}$ and number of cycles.

\begin{tabular}{ccl}
\hline \hline & \multicolumn{2}{c}{ Total length } \\
\cline { 2 - 3 } $\begin{array}{c}\text { Frequency } \\
(\mathrm{kHz})\end{array}$ & $\mathrm{ms}$ & cycles \\
\hline 0.5 & 10 & 5 \\
0.75 & 7 & 5.25 \\
1 & 5 & 5 \\
1.5 & 5 & 7.5 \\
2 & 5 & 10 \\
3 & 3.4 & 10.2 \\
4 & 2.5 & 10 \\
6 & 1.7 & 10.2 \\
8 & 1.25 & 10 \\
\hline \hline
\end{tabular}

\section{B. Apparatus and stimuli} 1. OAES

For the OAE recordings, the subjects were seated in a comfortable chair in an IEC 268-13 compliant sound insulated booth. Each recording session lasted around $45 \mathrm{~min}$ and the responses were recorded during three different sessions. The stimuli used for the OAE experiment were clicks and TBs repeated at a rate of $25 / \mathrm{s}$. Clicks were approximately $113 \mu$ s long and were presented 4000 times at 56 and $66 \mathrm{~dB}$ pe (peak equivalent) SPL. TBs at $0.5,0.75,1,2,3,4$, 6 , and $8 \mathrm{kHz}$ were presented 4000 times at $66 \mathrm{~dB}$ pe SPL, chosen to be comparable with historical studies. A lower level would compromise the comparison with ABR, whose waves are difficult to detect. At higher levels, the cochlear amplifier is not as active and the relative size of the OAEs is therefore lower (Kemp, 2002). It should also be noted that $66 \mathrm{~dB}$ pe SPL is relatively high so there will be some spread of excitation. Therefore, the OAE and ABR may originate from a larger area on the $\mathrm{BM}$. However, this was a necessary trade-off in the experimental design. TB frequencies ranging from 0.5 to $8 \mathrm{kHz}$ were used and their durations ranged between 10 and $1.25 \mathrm{~ms}$ (see Table I). The experiment was repeated three times for each subject to get a measure of intra-subject variability.

\section{Brainstem responses}

For the TB evoked ABR (TBABR) recordings, the subjects were laid down on a clinical couch in the same booth as for the OAE recordings. Subjects were encouraged to sleep during the experiment to reduce electrophysiological background noise. The ABR recordings were collected during three sessions, lasting about $2 \mathrm{~h}$ each. The same TB stimuli as in the OAE experiment were used in the ABR experiment. However, the number of averages varied with center frequencies for ABR. A preliminary experiment showed that wave $\mathrm{V}$ could be detected using fewer averages for high frequencies, due to the stronger signal strength at these frequencies. The lower frequencies $(0.5,0.75$, and $1 \mathrm{kHz})$ were repeated 8000 times, the middle frequencies $(1.5,2$, and $3 \mathrm{kHz}) 4000$ times, and the higher frequencies $(4,6$, and $8 \mathrm{kHz}) 3000$ times for each run.

The choice of the stimuli was inspired by the experiments from Norton and Neely (1987) and Şerbetçioğlu and
Parker (1999). They were generated following the standard IEC 645-3 on short-duration test signals. These durations represent a trade-off between having an equal number of cycles for all frequencies and a relative narrow spread in their spectrum. The organization of frequency along the cochlear partition is roughly logarithmic and TBs with a fixed number of cycles result in uniform energy splatter in logfrequency. The stimulus rise time is responsible for the simultaneous neural activation leading to the brainstem responses (Suzuki and Horiuchi, 1981) and to obtain a detectable ABR. A sharp stimulus onset (i.e., a short rise time) produces a large amount of synchronized neural activity, but also decreases the frequency specificity of the stimulus. Rise times for frequencies of $2 \mathrm{kHz}$ and above include approximately 5 cycles and therefore ranged from 2.5 to $1.25 \mathrm{~ms}$. Below $2 \mathrm{kHz}$ it was felt that the reduced energy spread, by keeping a fixed number of cycles, would make it almost impossible to record a wave- $\mathrm{V}$ response. Therefore, a compromise was struck, similar to Gorga et al. (1988), between the need for rapid stimulus onsets and reduced energy spread in the choice of rise time. The rise times were reduced to 3.25 at $1.5 \mathrm{kHz}$ and approximately 2.5 in the $0.5-1.0 \mathrm{kHz}$ range.

\section{Procedure}

The stimuli were produced in MATLAB, and then sent to a D/A converter (RME ADI8-Pro). The analog signal was transmitted to a programmable attenuator (TDT PA5) and a headphone driver (TDT HB7) and finally to the insert earphone ER-2. The stimuli were calibrated using an ear mould simulator (B\&K DB 0370) connected to an IEC 711 coupler $(B \& K$ 4157) and a B\&K 2607 sound level meter.

\section{OAES}

The TBOAEs were recorded in the right ear for most of the subjects but in the case of a blocked EC, the left ear was chosen (two subjects only). Studies have shown that the two ears of a subject presented very similar TEOAEs (Jedrzejczak et al., 2005; Moleti et al., 2008); therefore, the choice of ear should have no influence. The TBOAEs were recorded with an ER-10B low-noise microphone (Etymotic Research), band-pass filtered $0.15-16 \mathrm{kHz}$ with an analog filter (KrohnHite 3750), digitalized with the RME A/D converter and, finally, stored on a PC for off-line analysis. Both stimulus generation and response measurement were controlled via MATLAB.

\section{Brainstem responses}

For the TBABR, the responses were recorded using the Synamps I EEG amplifier and the data saved for off-line analysis. Despite attempts to shield the transducers, some electrical artifact signal due to the stimulus could be recorded in the response. However, an alternating polarity procedure (Gorga et al., 1988) was used to reduce this. It has previously been shown that this also leads to higher amplitude of the wave V, making its detection easier (Foxe and Stapells, 1993; Schönweiler et al., 2005). A repetition rate of $24.5 \mathrm{~Hz}$ was used. Studies have shown that the amplitude of 
TABLE II. Table of individual mean EC impulse response lengths and standard deviations.

\begin{tabular}{cccccccccccc}
\hline \hline Subject & 3 & 4 & 5 & 6 & 7 & 9 & 10 & 11 & 12 & 15 & 16 \\
$\mu_{\tau_{\mathrm{EC}}}(\mathrm{ms})$ & 3.1 & 4.2 & 4.1 & 3.6 & 2.2 & 2.9 & 4.3 & 4.3 & 2.1 & 4.0 & 3.9 \\
$\sigma_{\tau_{\mathrm{EC}}}(\mathrm{ms})$ & 0.1 & 0.1 & 0.2 & 0.3 & 1.6 & 0.4 & 0.2 & 0.2 & 0.2 & 0.3 & 0.1 \\
\hline \hline
\end{tabular}

the responses remains unaffected by repetition rates up to 35/s (Stapells and Picton, 1981) and the latency is stable for a large range of repetition rates (Burkard and Secor, 2002). The ground electrode was placed on the subjects' forehead, the reference electrode was placed at the vertex $[\mathrm{Cz}$ in the 10-20 electrode system, Jasper (1958)], and the remaining electrode was placed at the ipsilateral mastoid. The impedance of each electrode was maintained below $5 \mathrm{k} \Omega$. The order of presentation of the stimuli was randomized for each subject.

\section{Off-line data analysis}

\section{1. $O A E$ artefact rejection}

In order to reject epochs containing a great amount of noise due to subject movement or swallowing, artifact rejection was applied. Averages contaminated by external noise can be detected due to the presence of sudden high amplitude signals, the averages were ranked according to their maximum amplitude, and $10 \%$ of them were discarded.

\section{OAE detection method}

The detection of the OAE onset is difficult due to early components in the recorded pressure attributable to the transducer response and the EC (Stover and Norton, 1993). In previous studies, the separation between linear and nonlinear reflections has been identical for all subjects (Kemp, 1978; Norton and Neely, 1987; Keefe, 1998; Şerbetçigğlu and Parker, 1999; Jedrzejczak et al., 2005), by assuming that all have identical idealized ECs and middle ears. The paradigm used in the present study tries to determine the OAE onset in each subject separately. This method is based on the separation, in time, between the reflections occurring in the EC and the signal originating in the cochlea. This separation is made, on the one hand, by calculating the impulse response of the subject's EC and, on the other hand, by detecting the peak attributed to the OAE.

In order to calculate the impulse response of the subject's EC, two clicks are presented at two levels, 56 and $66 \mathrm{~dB}$ pe SPL, both sufficiently high to be in the compressive regions of the OAE input-output function. These transient responses can be considered as being composed of two components; the first is the EC and transducer response linearly scaled with input level; the second is the OAE compressively scaled with input level. The recorded transient responses are then normalized so that the peak absolute amplitude is set to unity. By normalizing the responses, the linear EC component in each should be identical within the noise floor of the recording. The OAE components on the other hand will differ due to the compressive input-output function. After normalization, the OAE component for the $66 \mathrm{~dB}$ pe SPL input level will be smaller relative to the OAE component for the $56 \mathrm{~dB}$ pe SPL excitation level. Thus, the region where these two transient response curves diverge indicates where the OAE dominates the time series. The EC impulse response estimate, $\tilde{h}_{\mathrm{EC}}(\tau)$, is thus defined as the normalized transient response up to the point the OAE response dominates, obtained via visual inspection for each subject. Table II gives the mean (across the three repeat runs) and standard deviation of the $\tilde{h}_{\mathrm{EC}}(\tau)$ durations for each subject.

Similarly, for the TB stimuli, it is assumed that the response recorded is composed of EC and OAE components, $p(t)=p_{\mathrm{EC}}(t)+p_{\mathrm{OAE}}(t)$. However, the low-level tail end of the $p_{\mathrm{EC}}(t)$ component overlaps with the start of the $p_{\mathrm{OAE}}(t)$. This OAE onset ambiguity is worse for TB stimuli as they are less well defined in time than a delta function or impulse. In order to obtain an estimate for the EC component, the estimated impulse response obtained from the transient analysis can be used and convolved with the TB stimuli to yield

$$
\tilde{p}_{\mathrm{EC}}(t)=\int \tilde{h}_{\mathrm{EC}}(\tau) x_{\mathrm{stim}}(t-\tau) d \tau,
$$

where $\tilde{p}_{\mathrm{EC}}(t)$ is the estimated EC pressure component due to the TB stimulus, $x_{\text {stim }}(t)$. Thus, to obtain the OAE latency a comparison is made between the stimulus onset and the peak of the OAE response to the TB, using the two time series, the recorded pressure due to the TB stimuli, $p(t)$, and the estimated pressure component due to the EC and transducers, $\tilde{p}_{\mathrm{EC}}(t)$. It is beneficial to restrict the analysis to a narrow frequency range around the TB center frequency, to remove any ambiguous components not directly evoked by the stimulus. One approach to narrowband analysis of the signals is via non-parametric time-frequency analysis, i.e., a simple linear spectrogram or a quadratic time-frequency representation such as the Wigner-Ville or related distributions (Cheng, 1995; Hatzopoulos et al., 2000; Konrad-Martin and Keefe 2003) or even a wavelet based method (Wit et al., 1994; Tognola et al., 1997; Moleti et al., 2005; Sisto and Moleti, 2007). These methods each have their advantages and disadvantages which will not be discussed here. In the present study, a parametric method of signal analysis was adopted from Long and Talmadge (1997), where an underlying model of the data is assumed. A least-squares fit was made to the following signal model:

$$
y=a \cos ((\omega+\Delta \omega) t)+b \sin ((\omega+\Delta \omega) t),
$$

where $\omega$ is the expected frequency of the signal (TB center frequency), and $\Delta \omega$ is a small offset around $\omega$ (where typically $\Delta \omega \ll 1)$, allowing detection despite small changes in frequency. This method uses a least-squares fitting procedure to obtain the unknown parameters $a, b$, and $\Delta \omega$, and allows the calculations of the instantaneous amplitude, $A(t)$ $=\sqrt{a^{2}(t)+b^{2}(t)}$, or envelope of the signal (see Long and Talmadge, 1997). 


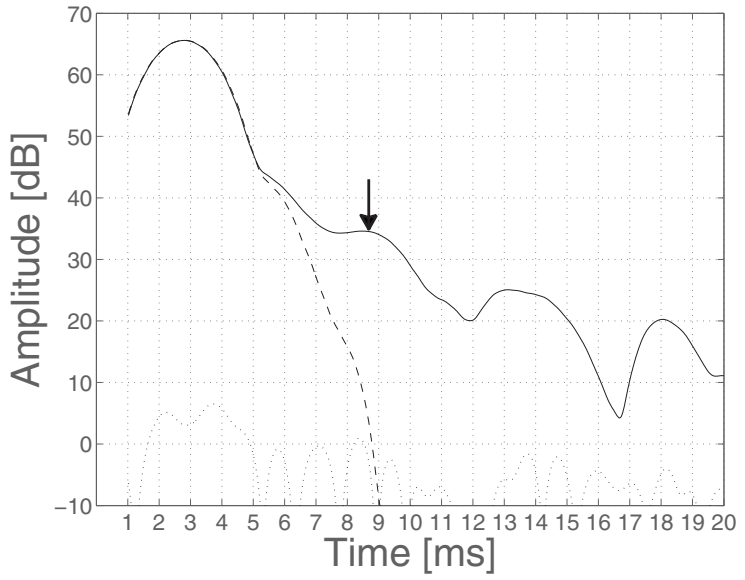

(a)

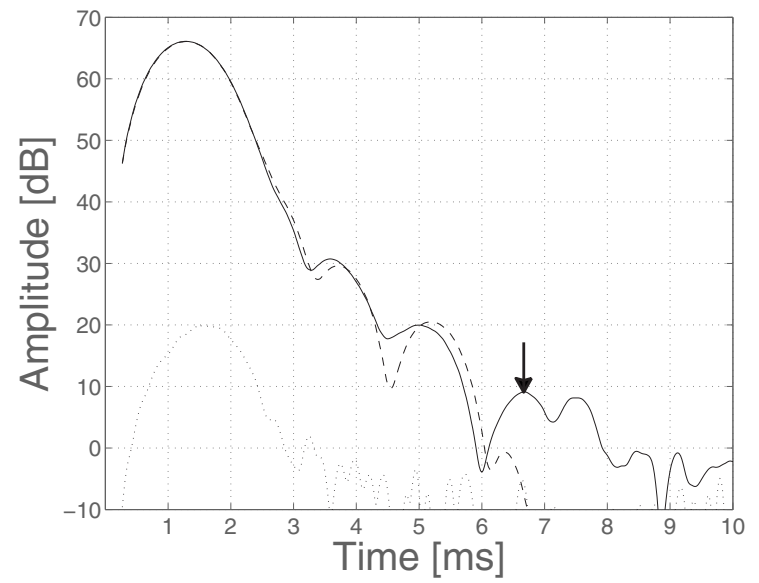

(b)

FIG. 1. Envelopes of the estimated EC and transducer signals (dashed curve), noise residue (dotted curve), and of the OAE response (solid curve) for (a) a $1 \mathrm{kHz}$ TB and (b) a $4 \mathrm{kHz}$ TB. The identified first peak attributed to the TBOAE is indicated by the arrow. Both these examples are from the same illustrative subject.

The least mean square fitting procedure therefore obtains the recorded signals envelope variation centered around the frequency of interest. Both the recorded pressure, $p(t)$, and the EC and transducer estimate, $\widetilde{p}_{\mathrm{EC}}(t)$, are processed through this algorithm, thus allowing detection of the first peak in $p(t)$ after the EC and transducer component has died away. An example is shown in Fig. 1(a) for a $1 \mathrm{kHz}$ TB and in Fig. 1(b) for a $4 \mathrm{kHz}$ TB. The dashed curves indicate the estimated EC component and the solid curve the recorded pressure. An arrow indicates the location of the OAE peak used to define latency. In order to label the OAE onset, the burst of energy envelope was required to be $6 \mathrm{~dB}$ above both the noise floor and the estimated EC component. In Fig. 1(a), the OAE delay, $\tau_{\mathrm{OAE}}$, was found to be $8.7 \mathrm{~ms}$ where the EC component had sufficiently decayed away from the recorded TB response. The noise floor in this case was around $35 \mathrm{~dB}$ below the OAE. The $4 \mathrm{kHz}$ delay in Fig. 1(b) was labeled as $6.6 \mathrm{~ms}$. In this case, the OAE component was only $12 \mathrm{~dB}$ above the noise floor. Typically, for the higher frequency TB stimuli, the reduced SNR made labeling more difficult and occasionally impossible for some subjects.

There are a number of peaks and dips appearing in the recorded signal, each with different levels and latencies. The expected level of the OAEs is about $45 \mathrm{~dB}$ below the peak stimulus level (Wilson, 1980a; Wit and Ritsma, 1980). The OAE latency was defined, in the present study, as the time between the onset of the stimulus and the peak local maximum detected as an OAE, following Şerbetçioğlu and Parker (1999). The method used in the present study does not allow to detect the OAE burst with a 100\% certainty. Although best efforts were made to provide a reliable tool, the OAE onset ambiguity is not fully solved. This ambiguity seems to be inherent to any TEOAE recordings.

\section{ABR off-line analysis}

The ABR recordings were first epoched and averaged using an iterative weighted-averaging algorithm (Riedel et al., 2001). The responses were then filtered, between 0.1 and $1.5 \mathrm{kHz}$. Wave $\mathrm{V}$ peaks and latencies were determined assuming a sufficiently high signal to noise ratio and good repeatability across the three repeated runs.

\section{RESULTS}

\section{A. Experimental data}

The individual OAE delay, $\tau_{\mathrm{OAE}}$, and BM delay estimated from $\mathrm{ABR}, \hat{\tau}_{\mathrm{BM}}^{(\mathrm{ABR})}$, are shown in the left and right panel of Fig. 2, respectively. As expected, these two delays show exponentially decreasing delays as a function of frequency. The detection of wave $\mathrm{V}$ at low frequencies proved to be impossible for some subjects, despite the high number of averages (8000). This problem at low frequencies has been pointed out in many previous studies (e.g., Stapells, 1994; Stürzebecher et al., 2006) and could be due to the lower speed of the traveling wave in the low-frequency region of the cochlea compared to the basal part. With a lower
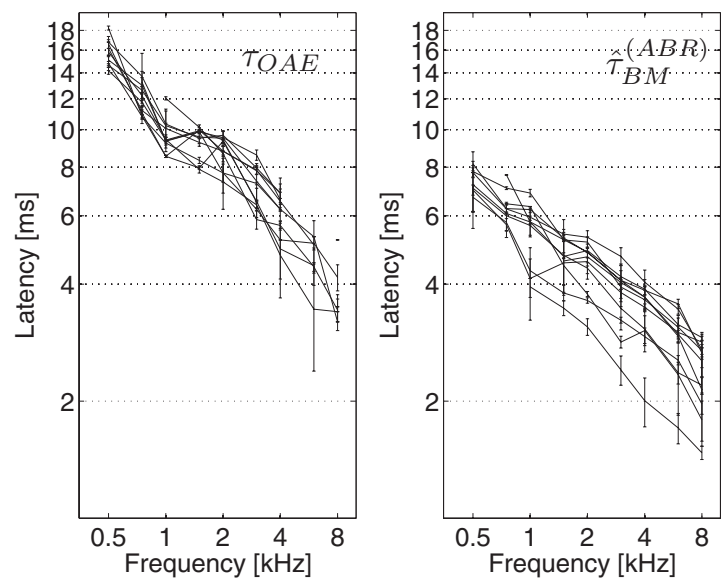

FIG. 2. The OAE and BM latency estimates for the 11 subjects are shown in the left and right panels, respectively. The OAE latency is defined as the time between stimulus onset and the peak of the OAE burst. The BM latency estimate, $\hat{\tau}_{\mathrm{BM}}^{(\mathrm{ABR})}$, is calculated following Eq. (2). The error bars represent \pm 1 std. 
TABLE III. Inter-subject variability for $\tau_{\mathrm{OAE}}$ and $\hat{\tau}_{\mathrm{BM}}^{(\mathrm{ABR})}$ across frequency and given in percentage. The percentage is calculated as the ratio between the standard deviation and the mean value.

\begin{tabular}{lccccccccc}
\hline \hline Freq. (kHz) & 0.5 & 0.75 & 1 & 1.5 & 2 & 3 & 4 & 6 & 8 \\
$\tau_{\mathrm{OAE}}$ var. (\%) & 8.2 & 8.9 & 10.1 & 9.0 & 9.4 & 12.3 & 13.3 & 14.7 & 18.8 \\
$\hat{\tau}_{\mathrm{BM}}^{\mathrm{ABR}}$ & 10.2 & 10.8 & 16.9 & 10.9 & 10.9 & 12.3 & 12.4 & 14.4 & 14.0 \\
\hline \hline
\end{tabular}

velocity, adjacent nerves fire with a certain delay, leading to asynchronous neural activity, and thus a lower amplitude of the ABR. An increase in the stimulus level would produce a more detectable wave $\mathrm{V}$ at $0.5 \mathrm{kHz}$, due to a greater discharge of the neurons (Gorga et al., 1988). However, the stimulus level of the TBABR measurements ( $66 \mathrm{~dB}$ pe SPL) was chosen according to the OAE experiment for better comparability. A level higher than $66 \mathrm{~dB}$ pe SPL in the OAE experiment would have resulted in a relatively lower response and to a reduced frequency specificity due to broader filter activation (Ruggero et al., 1997).

\section{B. Data analysis}

\section{Intra- and inter-subject variabilities}

For both measurement methods, the intra-subject variability of the latency is relatively small (error bars in Fig. 2). For OAEs (left panel), the maximum standard deviation is $2.01 \mathrm{~ms}$ for subject 10 at $0.75 \mathrm{kHz}$, which is rather small in comparison to the latency at that frequency (13.7 ms). However, this variability is only determined using three (at most) repeat measures and should therefore only be considered as a rough estimate. Some of the intra-subject variability could be due to the difficulty to assessing the true OAE onset, and not a component of the EC or middle ear response. Similarly, for ABR (right panel), the maximum standard deviation observed is $1.13 \mathrm{~ms}$ at $0.5 \mathrm{kHz}$ for subject 3 . The reproducibility of both these data sets is high and indicates that both techniques are reliable.

Figure 2 also demonstrates the inter-subject variability, which appears similar across frequency for both OAEs and ABRs. At $0.5 \mathrm{kHz}$, it is 1.29 and $0.72 \mathrm{~ms}$ for OAEs and $\mathrm{ABR}$, respectively, and at $8 \mathrm{kHz}$ it is 0.71 and $0.33 \mathrm{~ms}$. It is necessary to consider the relative standard deviation with respect to the magnitude of the latency estimates at each frequency. The inter-subject variability, in percentage, is calculated as the ratio between the standard deviation and the mean value. Percentages are used in order to compare with the results from Norton and Neely (1987). The relative variabilities found in this study, listed in Table III, are of the same order as the ones found by Norton and Neely (1987). The relative variability of $\widetilde{\tau}_{\mathrm{BM}}^{(\mathrm{ABR})}$ varies between $10.2 \%$ and $16.9 \%$, with little systematic dependence on frequency. For $\tau_{\mathrm{OAE}}$, the range from $8.2 \%$ to $18.8 \%$ is slightly larger. However, the variability of $\tau_{\mathrm{OAE}}$ is larger at higher frequencies probably due to the difficulties in correctly labeling the OAE onset. It is seen that the latency estimates differ slightly more between subjects for OAE than for ABR. On the cochlear level, subjects can indeed reflect different cochlear filtering properties which may variably affect the traveling wave. They may also have different hearing thresholds across the audible frequency range (Don et al., 1994). These differences affect, however, both the OAE and ABR inter-subject variability. In the case of ABR, variabilities also occur at a neuronal level, due to different head sizes or gender (Hall et al., 1988; Don et al., 1993; Burkard and Secor, 2002).

\section{Ratio of delay estimates}

Figure 3 (a) shows, the OAE delay, $\tau_{\mathrm{OAE}}$, and the derived $\mathrm{BM}$ delay from $\mathrm{ABR}$ estimates, $\hat{\tau}_{\mathrm{BM}}^{(\mathrm{ABR})}$, plotted together for an illustrative subject. Figure 3(b) shows the same for the mean across subjects. The delay-frequency relations for both $\tau_{\mathrm{OAE}}$ and $2 \hat{\tau}_{\mathrm{BM}}^{(\mathrm{ABR})}$ can be modeled as a power law similar to Neely et al. (1988), where

$$
\tau \propto \beta f^{-\alpha} .
$$

Plotting this on a double-log axis will result in a straight line with slope $-\alpha$ and ordinate intercept $\beta$. The parameters $-\alpha$ and $\beta$ were found for both $\tau_{\mathrm{OAE}}$ and $2 \hat{\tau}_{\mathrm{BM}}^{(\mathrm{ABR})}$ for each subject and for the across subject mean using an unconstrained nonlinear optimization routine in MATLAB. The model fitting parameters are given in Table IV. For comparison, equivalent of Neely et al. (1988) to $\beta$ was 4.46, which is around half of the values obtained here. This is as expected as Neely et al. (1988) fitted this function to $\hat{\tau}_{\mathrm{BM}}^{(\mathrm{ABR})}$ instead of $2 \hat{\tau}_{\mathrm{BM}}^{(\mathrm{ABR})}$ as used in the present study and $\beta$ represents the ordinate intercept on a double-log plot. Multiplying $\hat{\tau}_{\mathrm{BM}}^{(\mathrm{ABR})}$ by 2 will have no effect on the slope $-\alpha$, where Neely et al. (1988) found a value of 0.413 which is comparable to those values found here in Table IV. The solid and dashed lines in Fig. 3(a) show the model best fit to $\tau_{\mathrm{OAE}}$ and $2 \hat{\tau}_{\mathrm{BM}}^{(\mathrm{ABR})}$ for one illustrative subject; Fig. 3(b) shows the comparison for the group mean values. If $\tau_{\mathrm{OAE}}$ and $2 \hat{\tau}_{\mathrm{BM}}^{(\mathrm{ABR})}$ would align then we could argue that the $\mathrm{OAE}$ is generated at the tonotopic resonant

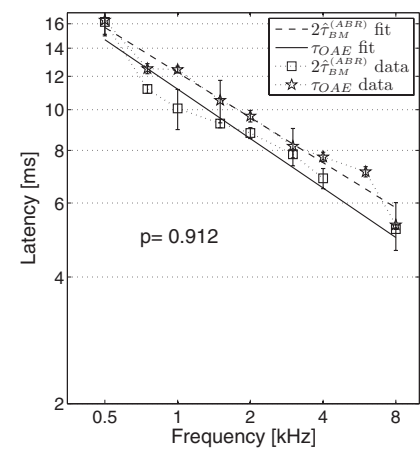

(a)

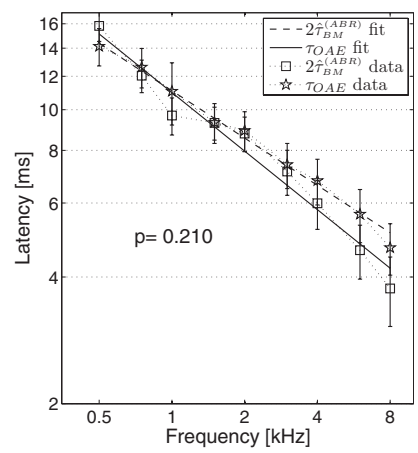

(b)
FIG. 3. Comparison between $\tau_{\mathrm{OAE}}$ (solid curve) and $2 \hat{\tau}_{\mathrm{BM}}^{(\mathrm{ABR})}$ (dashed curve) for (a) subject 10 and (b) mean across subjects. The data points are also plotted (symbols $\star$ and $\square$ ) and are connected by dotted lines. The solid and dashed lines represent the best fit to the data. The value of $p$ resulting from the two-way ANOVA test is indicated. For both the illustrative subject and the mean across subjects $p<0.05$, it is therefore considered that OAE and $\mathrm{ABR}$ are significantly different. 
TABLE IV. Delay power-law function fitting parameters for Eq. (5) across subject. Note the mean refers to the model fitted to the mean latency data and not the mean of the parameters shown.

\begin{tabular}{|c|c|c|c|c|}
\hline Subject & $\begin{array}{c}\alpha_{2 \hat{\tau}_{\mathrm{BM}}(\mathrm{ABR})} \\
\left(\mathrm{ms}^{2}\right)\end{array}$ & $\begin{array}{c}\beta_{2 \hat{\tau}_{\mathrm{BM}}^{(\mathrm{ABR})}} \\
\left(\mathrm{ms}^{2}\right)\end{array}$ & $\begin{array}{l}\alpha_{\tau_{\mathrm{OAE}}} \\
\left(\mathrm{ms}^{2}\right)\end{array}$ & $\beta_{\tau_{\mathrm{OAE}}}$ \\
\hline 3 & 0.42 & 9.76 & 0.57 & 10.85 \\
\hline 4 & 0.28 & 10.41 & 0.31 & 11.13 \\
\hline 5 & 0.48 & 8.16 & 0.42 & 11.04 \\
\hline 6 & 0.54 & 11.51 & 0.44 & 10.22 \\
\hline 7 & 0.45 & 12.77 & 0.57 & 10.40 \\
\hline 9 & 0.41 & 10.89 & 0.47 & 10.82 \\
\hline 10 & 0.36 & 12.25 & 0.39 & 11.19 \\
\hline 11 & 0.31 & 12.19 & 0.46 & 11.89 \\
\hline 12 & 0.34 & 12.55 & 0.59 & 12.35 \\
\hline 15 & 0.31 & 11.45 & 0.36 & 10.71 \\
\hline 16 & 0.31 & 10.43 & 0.42 & 11.08 \\
\hline Mean & 0.37 & 11.09 & 0.46 & 10.98 \\
\hline
\end{tabular}

place and propagates backwards via a reverse traveling wave. If they are parallel to each other, this would support a factor different from 2 since, on a log-log axis, a multiplication factor just shifts the curves up and down. To consolidate this visual analysis, a two-way analysis of variance (ANOVA) was carried out. It examines the effect of independent factors on the BM latency estimate. The independent factors are the frequency $(n=9)$ and the measurement technique $(n=2, \mathrm{ABR}$ or $\mathrm{OAE})$. The null hypothesis is as follows: The estimate of $\tau_{\mathrm{BM}}$ does not differ between techniques. Results are declared significant if the $p$-value is less than 0.05 and this would cast doubt on the null hypothesis.

The results of the ANOVA test ( $p$-values) for one exemplary subject and the mean across subjects are also presented in Figs. 3(a) and 3(b), respectively. For the exemplary subject, the slopes of the OAE delay and two times the ABR estimated BM delay, $\hat{\tau}_{\mathrm{BM}}^{(\mathrm{ABR})}$, do not differ significantly $(p$ $=0.912)$. In other words, the two techniques estimate the same rate of change of latency, i.e., the delay relation for $\tau_{\mathrm{OAE}}$ and $2 \hat{\tau}_{\mathrm{BM}}^{(\mathrm{ABR})}$ are statistically similar. Likewise, the slopes for the latencies averaged across subject [Fig. 3(b)] are the same, with the ANOVA test yielding a $p$-value of 0.210 . The ANOVA test was run on the 11 subjects and the $p$-values given in Table V. For 8 of the 11 subjects, it was found that $p>0.05$, implying that the slopes of $\tau_{\mathrm{OAE}}$ and $2 \hat{\tau}_{\mathrm{BM}}^{(\mathrm{ABR})}$ are not significantly different in most cases. This suggests that the OAE being generated by a tonotopically resonant place and propagating back via a reverse traveling wave would be supported by these results, since the data obtained in this study verify that $\tau_{\mathrm{OAE}} \approx 2 \hat{\tau}_{\mathrm{BM}}^{(\mathrm{ABR})}$ for most of the subjects.

It has been suggested (Narayan, 1991) that the return trip for OAEs is not the same as the forward traveling wave, i.e., that there is no retrograde wave and that OAEs might travel faster on their way back. Therefore, the factor 2 between $\tau_{\mathrm{OAE}}$ and $\hat{\tau}_{\mathrm{BM}}^{(\mathrm{ABR})}$ might not be correct. Shera and Guinan (2003) found factors relating $\tau_{\mathrm{BM}}$ to $\tau_{\mathrm{OAE}}$ of 1.7 and 1.6 for cats and guinea pigs using SFOAEs, respectively, and Moleti and Sisto (2008) found a factor of 2.08 \pm 0.19 in humans using TEOAEs. In the present study, this factor was
TABLE V. Statistical analysis results. Column 2 gives the subject dependent interpeak and synaptic delay means \pm 1 standard deviation, with the difference from that used by Moleti and Sisto (2008) given in parentheses. Column 3 gives the $p$-values from the ANOVA test with any significant results given in bold. The $p$-values in parentheses were obtained when using the fixed Moleti and Sisto (2008) interpeak and synaptic delay. Note the mean refers to the results for the mean latency data and not the mean of the parameters shown.

\begin{tabular}{ccc}
\hline \hline & $\begin{array}{c}2 \cdot \Delta_{\mathrm{III}-\mathrm{V}}+\tau_{\text {synaptic }} \\
\mathrm{ms} \pm 1 \text { s.d. } \\
(\mathrm{ms})\end{array}$ & $\begin{array}{c}\text { ANOVA } \\
p \text {-value }\end{array}$ \\
Subject & $4.40 \pm 0.12(0.6)$ & $0.168(0.450)$ \\
3 & $4.16 \pm 0.08(0.84)$ & $0.203(0.661)$ \\
4 & $5.16 \pm 0.24(-0.16)$ & $0.495(0.358)$ \\
5 & $4.96 \pm 0.08(0.04)$ & $0.971(0.923)$ \\
6 & $4.56 \pm 0.14(0.44)$ & $0.251(0.575)$ \\
7 & $4.40 \pm 0.18(0.60)$ & $0.947(0.386)$ \\
9 & $4.64 \pm 0.30(0.36)$ & $0.912(0.633)$ \\
10 & $4.14 \pm 0.22(0.85)$ & $\mathbf{0 . 0 1 6}(0.194)$ \\
11 & $4.20 \pm 0.18(0.80)$ & $0.286(0.974)$ \\
12 & $4.36 \pm 0.08(0.65)$ & $\mathbf{0 . 0 1 8}(\mathbf{0 . 0 4 4})$ \\
15 & $4.56 \pm 0.26(0.44)$ & $\mathbf{0 . 0 0 6}(\mathbf{0 . 0 2 2})$ \\
16 & $4.52 \pm 0.36(0.49)$ & $0.210(0.640)$ \\
Mean &
\end{tabular}

calculated from all $\tau_{\mathrm{OAE}}$ and $\hat{\tau}_{\mathrm{BM}}^{(\mathrm{ABR})}$ pairs (a total of 81 points) and found to be $1.92 \pm 0.42 \mathrm{~ms}$. This standard deviation is relatively high, and is probably dependent on outliers due to incorrect identification of the OAE onset. Figure 4 shows a histogram for all of the possible calculated ratios across the whole frequency range and all subjects. The light gray bars could be considered outliers from a more Gaussian distribution of ratios (darker gray bars). If the ratio were recalculated only on the dark gray subset of results, then it is found to be $1.85 \pm 0.21 \mathrm{~ms}$. However, as mentioned earlier, the factor between $\tau_{\mathrm{OAE}}$ and $\hat{\tau}_{\mathrm{BM}}^{(\mathrm{ABR})}$ just shifts the curves and their slopes are not affected. The present analysis therefore remains unaffected by the exact constant of proportionality between these two delays.

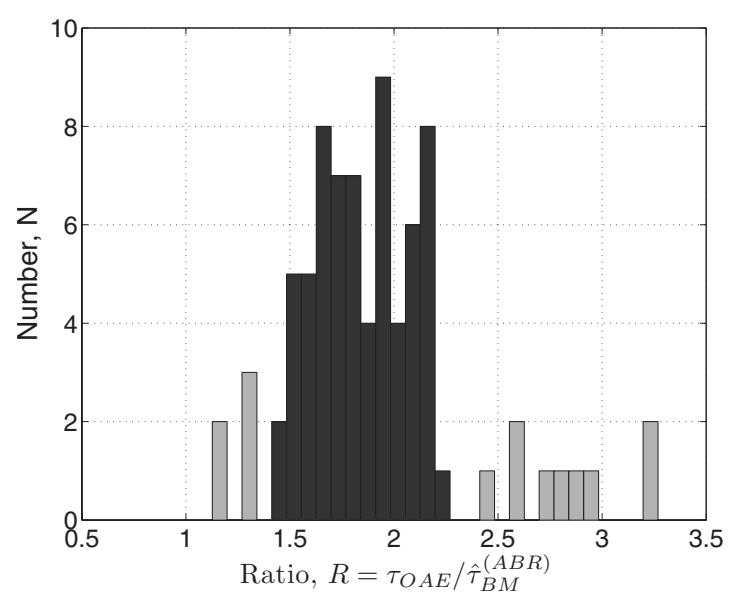

FIG. 4. Histogram of all calculated $\tau_{\mathrm{OAE}}$ to $\hat{\tau}_{\mathrm{BM}}^{(\mathrm{ABR})}$ ratios. Group shown in light gray represent potential outliers. 


\section{DISCUSSION}

\section{A. Revisiting the assumptions for the delay estimates 1. Role of middle-ear delay}

As discussed by Moleti and Sisto (2008) and Abdala and Keefe (2006), neglecting the middle-ear delay could lead to misinterpretation of the cochlear delay estimates based on OAE and ABR recordings. Puria (2003) made measurements of human middle ear forward and reverse acoustics, and discussed their implications for OAEs. In particular, he recorded the frequency dependent forward, backward, and round trip pressure gain and phase. This showed a significant bandpass type characteristic with frequency. Both delay estimates obtained in this study, $\tau_{\mathrm{OAE}}$ and $\hat{\tau}_{\mathrm{BM}}^{(\mathrm{ABR})}$ would be affected by the forward transmission. Puria (2003) showed this to be maximum around $0.9 \mathrm{kHz}$ with a slope of $-10.4 \mathrm{~dB} /$ oct below it and $-7.2 \mathrm{~dB} /$ oct above it. This implies a difference in effective stimulus level at the stapes. Cochlear tuning is lower at higher stimulus levels, thus introducing an additional dependence on frequency for the stimuli used in this experiment. It is difficult to quantify this difference; however, it should be the same for both delay estimates $\tau_{\mathrm{OAE}}$ and $\hat{\tau}_{\mathrm{BM}}^{\mathrm{ABR})}$. ABR forward latency of Neely et al. (1988) was well represented by the function, also used here, $\tau_{\mathrm{BM}} \propto \beta f^{-\alpha}$ across $0.25-8 \mathrm{kHz}$. Therefore, this powerlaw relation appears independent of middle-ear filtering.

Additional to the frequency-dependent delay associated with different excitation levels, Puria (2003) recorded the phase of the forward and reverse transmission paths, leading to an accumulated group delay. Within the range $2-6 \mathrm{kHz}$, the phase angle decreases with increasing frequency for the forward transmission path with a slope of $-73^{\circ} /$ oct, with the reverse transmission path having a slope of $-105^{\circ} /$ oct. If this is converted to group delay, this would imply approximately $0.08 \mathrm{~ms}$ in forward and $0.12 \mathrm{~ms}$ in the reverse directions. Thus, $\tau_{\mathrm{OAE}}$ would have a delay of $0.2 \mathrm{~ms}$ attributed to middle-ear transmission (forward and reverse).

Multiplying the ABR delay estimate, $\hat{\tau}_{\mathrm{BM}}^{(\mathrm{ABR})}$, by two would result in a middle-ear round trip delay of twice the forward delay, $0.16 \mathrm{~ms}$. Thus, in the frequency region $2-6 \mathrm{kHz}$ there is an additional transmission delay expected in the OAE over the ABR delay estimates due to the round trip through the middle ear of $0.04 \mathrm{~ms}$. However, this is considered here to be small relative to the existing experimental error, and is therefore thought to be negligible.

\section{Effects of neural and synaptic delays}

In the present study, the cochlear delay estimate, $\hat{\tau}_{\mathrm{BM}}^{(\mathrm{ABR})}$, was made using individual estimates of interpeak or neural delays, i.e., it was assumed that $\Delta_{\mathrm{I}-\mathrm{V}}=2 \cdot \Delta_{\mathrm{III}-\mathrm{V}}$, as also used by Don and Eggermont (1978); Eggermont and Don (1980) as well as Don and Kwong (2002). The wave-III to wave-V delays were recorded in all subjects tested for frequencies in the range $2-8 \mathrm{kHz}$. Below $2 \mathrm{kHz}$ it was felt that wave-III could not be consistently recorded. The averaged neural and synaptic delays for each subject, as well as a grand mean, are shown in Table $\mathrm{V}$ alongside the standard deviation. The synaptic delay was assumed here to be constant and $1 \mathrm{~ms}$.
Another approach for dealing with the unknown neural delay is to assume a constant delay across subjects. For example, Don et al. (1993) used a fixed interpeak delay of $4.0 \mathrm{~ms}$ for males and $3.8 \mathrm{~ms}$ for females. Moleti and Sisto (2008) subtracted a constant offset of $4.2 \mathrm{~ms}$ for the neural delay and assumed a $0.8 \mathrm{~ms}$ synaptic delay. The grand average delay used in this study was $4.52 \mathrm{~ms}$, which would under-predict the estimate obtained from $5.0 \mathrm{~ms}$ by $0.48 \mathrm{~ms}$ of Moleti and Sisto (2008). However, this offset or difference across subjects ranges from $0.86 \mathrm{~ms}$ under- to $0.16 \mathrm{~ms}$ overprediction relative to Moleti and Sisto (2008)'s fixed delay. The results reported here, using a subject-dependent delay, would therefore tend to under-predict the estimate $\hat{\tau}_{\mathrm{BM}}^{(\mathrm{ABR})}$ relative to method of Moleti and Sisto (2008).

The statistical analysis used in this study was based on comparing the evolution of delay with frequency. A constant offset of delay would thus change the slope of $\hat{\tau}_{\mathrm{BM}}^{\mathrm{ABR})}$ when plotted on a double-log plot. This could potentially alter the conclusions, as the assumptions on Eq. (2) would more significantly affect the high-frequency delay estimates, due to the shorter delays in this region relative to the offset. Table $\mathrm{V}$ presents in round brackets the results of the ANOVA test applied when using the fixed neural delay from Moleti and Sisto (2008). It can be seen that 9 of the 11 subjects demonstrated no significant difference in their slopes. It is apparent that this ANOVA test is sensitive to the assumption of how to model neural delay. However, both the ABR and OAE methods tend to give the same evolution of latency with frequency.

\section{B. Effect of TB rise time on wave-V latency}

The TBs used to measure OAEs and ABRs were inspired by the experiment of Neely et al. (1988), who used different rise times across frequency in order to have the same width of BM excitation for each stimulus. The choice for different absolute rise times for different frequencies has recently been criticized by Ruggero and Temchin (2007) who claimed that this difference adds a supplementary delay to $\tau_{\text {wave } \mathrm{V}}$, which would yield a change in the latency slope function. They argued that identical TB rise times are necessary to have the synchronous neural firing occurring at the same time for all the stimuli. Neely et al. (1988) used TBs with onset-ramp durations that decreased as a function of increasing frequency (e.g., $4 \mathrm{~ms}$ for 0.25 and $0.5 \mathrm{kHz}, 2 \mathrm{~ms}$ for $1-2 \mathrm{kHz}, 1.4 \mathrm{~ms}$ for $3 \mathrm{kHz}, 1 \mathrm{~ms}$ for $4 \mathrm{kHz}$, and $0.5 \mathrm{~ms}$ for $8 \mathrm{kHz}$ ). Ruggero and Temchin (2007) argued that this should artificially produce delays that increase as stimulus frequency decreases, based on the study on auditory nerve fiber first spike firing by Heil and Irvine (1997). Ruggero and Temchin (2007) cited Heil and Irvine's (1997) Figs. 2E and $3 \mathrm{E}$, that for a $30 \mathrm{~dB}$ SPL tone, increasing the ramp duration from 1.7 to $4.2 \mathrm{~ms}$ can increase the first-spike latency by $1.5-3 \mathrm{~ms}$. This calls into question some of the findings for Neely et al. (1988) at low excitation levels. However, citing again Heil and Irvine's (1997) Figs. 2E and 3E, this time for a $70 \mathrm{~dB}$ SPL tone, the first-spike latency increase is reduced to the order of $0.3 \mathrm{~ms}$. Therefore, at the moderate excitation levels used in the present study, the role of TB rise time 
should be minimal and below the range of experimental precision. It is thus argued here that this aspect does not alter the conclusions of the current study.

\section{Implications for OAE generation mechanisms}

A discussion on the OAE generation mechanisms is made as these are still under debate and the subject of controversy in the literature (Ren and Nuttall, 2006; Shera et al., 2007; Ruggero and Temchin, 2007; Dong and Olson, 2008; Shera et al., 2008). The present study suggests that OAE delay, $\tau_{\mathrm{OAE}}$, fits relatively well with the idea that OAEs are generated at the tonotopic site and propagate backwards to the base as a traveling wave. The approximate ratio of $\mathrm{OAE}$ delay, $\tau_{\mathrm{OAE}}$, to the $\mathrm{BM}$ delay estimated from $\mathrm{ABRs}, \hat{\tau}_{\mathrm{BM}}^{\mathrm{ABR})}$, was found to be $1.92 \pm 0.42 \mathrm{~ms}$ or $1.85 \pm 0.21 \mathrm{~ms}$ if one could justify the removal of the outliers. This is also consistent with the approximate CRF theory prediction of a factor of 2. Moleti and Sisto (2008) found a factor of $2.08 \pm 0.19$ in humans; however, the study used TEOAE results compared with historical ABR recordings evoked from both TBs and derived band techniques. In the present studies, the same subjects were tested with identical stimulus types for both $\mathrm{ABR}$ and $\mathrm{OAE}$ recordings. The mean ratios were similar for both studies; however, the present study demonstrated a rather large variability of $0.42 \mathrm{~ms}$. This is possibly due to the methods employed here to identify the OAE onset burst, where mislabeling would lead to large outliers.

The present results are in agreement with previous animal studies that showed reasonable agreement with the CRF prediction at high frequencies (Shera and Guinan, 2003; Siegel et al., 2005). Shera and Guinan (2003) compared SFOAE group delays measured in cats and guinea pigs with BM mechanical transfer functions taken from the literature. They found that the relation $\tau_{\mathrm{OAE}} \approx 2 \tau_{\mathrm{BM}}$ holds for the basal part of the cochlea only (i.e., above $4 \mathrm{kHz}$ ). Siegel et al. (2005) tested the hypothesis that SFOAE group delay was twice that of BM delay. They estimated the BM delay by using Wienerkernel analysis of responses to noise of auditory nerve-fibers in chinchillas. Siegel et al. (2005) demonstrated that SFOAE group delays were slightly below that predicted with the simplified assumption of coherent reflection at frequencies above $4 \mathrm{kHz}$, i.e., $\tau_{\mathrm{SFOAE}} \neq 2 \tau_{\mathrm{BMgroup}}$. However, they claimed that the data are indeed compatible with the hypothesis of SFOAE propagation to the stapes via fluid coupling (compression-wave) or via reverse BM traveling wave with speeds corresponding to the signal-front delays rather than group delays of forward waves. These results contradict the CRF prediction, but not the reflection mechanism itself. Shera et al. (2008) offered a different physical argument why the factor between $\tau_{\mathrm{OAE}}$ and $\tau_{\mathrm{BM}}$ is below 2 for frequencies above $4 \mathrm{kHz}$. They used a semi-analytic form (developed by Shera et al., 2005) of the coherent reflection model, and tested model predictions tailored for chinchilla cochleae against recorded SFOAE delays. The model predicts SFOAE delays, $\hat{\tau}_{\mathrm{SFOAE}}$, corresponding to the round trip delays for pressure difference waves, rather than BM velocity traveling waves (or alternatively "signal-front" delay). Revised prediction of Shera et al. (2005) for CRF theory is summarized as
$\hat{\tau}_{\mathrm{SFOAE}} \approx 2\left(1-\hat{\tau}_{\mathrm{BM}} / \hat{\tau}_{k}\right)$, where $\hat{\tau}_{k}$ is a positive delay, empirically determined by Shera et al. (2008) to be approximately $10-20 \%$ of $\hat{\tau}_{\mathrm{BM}}$. This delay arises from the complex relation between the transportation pressure across the cochlear partition and the BM velocity or travelling wave pattern. As $\hat{\tau}_{k}$ is positive, the predicted delay ratio between BM velocity delay and SFOAE delay is typically less than 2 . The data shown in the present study would be consistent with this prediction. Shera and Guinan (2003), Siegel et al. (2005), and Shera et al. $(2008,2005)$ made use of SFOAEs and obtained delay estimates using phase-gradient delays. As pointed out by Moleti and Sisto (2008) and Sisto et al. (2007), the interpretation of the SFOAE phase-gradient delays in terms of cochlear transmission delays is model dependent, due to the necessary simplifying assumptions to obtain a tractable model. Therefore, it is not possible to enter the debate of OAE generation mechanisms in the present study, other than to say that the data seem to align well with the general linear CRF theory model predictions at higher frequencies.

For frequencies below $4 \mathrm{kHz}$, Siegel et al. (2005) argued that the errors of CRF-theory prediction are much greater. They argued that CRF could not account for their data and that a different model of reverse energy propagating backwards outside the cochlea via a compression wave was necessary, as first suggested by Wilson (1980b). This was also suggested by Ren (2004), who used scanning laser interferometry to detect forward-traveling waves in gerbil cochleae, but failed to provide any evidence for backwardtraveling waves. Differences in experimental paradigms and species could be the cause of the present study contradicting the conclusions of Siegel et al. (2005), where the present study would tend to support CRF theory throughout the range of frequencies tested. However, it should be noted that even though there is good experimental agreement between SFOAE and TEOAE, when stimulus intensity is in bandwidth-compensated SPL (cSPL) (Kalluri and Shera, 2007), the levels used in this study ranged from $42-58 \mathrm{~dB}$ cSPL as TB frequency drops from 8 to $0.5 \mathrm{kHz}$ systematically. It is not clear what this level variation and the effects of spread of excitation may do for the OAE model predictions on delay, so one should be careful making a direct comparison to the historical studies reported in this section. Overall, these data from the present study seem to support the coherent reflection filtering theory within the experimental limitations.

\section{SUMMARY AND CONCLUSION}

This study estimated cochlear delays in humans using both OAEs and ABRs, evoked from TBs in the same subjects. It was shown that latency estimates can be reliably obtained from both methods. This study demonstrated that the ratio of $\mathrm{OAE}$ delay, $\tau_{\mathrm{OAE}}$, to the $\mathrm{BM}$ delay estimated from ABRs, $\hat{\tau}_{\mathrm{BM}}^{(\mathrm{ABR})}$, to be $1.92 \pm 0.42 \mathrm{~ms}$, which is in line with the argument that OAEs are generated at a tonotopic place and propagate backward to the base as a reverse traveling wave, with the same speed as that of the forward wave. 
This is further supported by the similar variation of delay with frequency between both the OAE delay, $\tau_{\mathrm{OAE}}$, and the $\mathrm{BM}$ delay estimated from ABRs, $\hat{\tau}_{\mathrm{BM}}^{\mathrm{ABR})}$.

\section{ACKNOWLEDGMENTS}

The OAE recording software was developed in collaboration with Manfred Mauermann at the Carl von Ossietzky Universität in Oldenburg, Germany. This project was jointly funded by GN Resound A/S, Oticon A/S, and Widex A/S and carried out by Gilles Pigasse as part of his doctoral studies.

Abdala, C., and Keefe, D. (2006). "Effects of middle-ear immaturity on distortion product otoacoustic emission suppression tuning in infant ears," J. Acoust. Soc. Am. 120, 3832-3842.

Burkard, R., and Secor, C. (2002). "Overview of auditory evoked potential," in Handbook of Clinical Audiology, edited by J. Katz (Lippincott, Williams, and Wilkins, Philadelphia, PA), Chap. 14, pp. 233-248.

Cheng, J. (1995). "Time-frequency analysis of transient evoked otoacoustic emissions via smoothed pseudo Wigner distribution," Scand. Audiol. 24, 91-96.

Don, M., and Eggermont, J. J. (1978). "Analysis of the click-evoked brainstem potentials in man using high-pass noise masking," J. Acoust. Soc. Am. 63, 1084-1092.

Don, M., and Kwong, B. (2002). "Auditory brainstem response: Differential diagnosis," in Handbook of Clinical Audiology, edited by J. Katz (Lippincott, Williams, and Wilkins, Philadelphia, PA), Chap. 16, pp. 274-297.

Don, M., Ponton, C. W., Eggermont, J. J., and Kwong, B. (1998). "The effects of sensory hearing loss on cochlear filter times estimated from auditory brainstem response latencies," J. Acoust. Soc. Am. 104, 2280 2289.

Don, M., Ponton, C. W., Eggermont, J. J., and Masuda, A. (1993). “Gender differences in cochlear response time: An explanation for gender amplitude differences in the unmasked auditory brain-stem response," J. Acoust. Soc. Am. 94, 2135-2148.

Don, M., Ponton, C. W., Eggermont, J. J., and Masuda, A. (1994). “Auditory brainstem response peak amplitude variability reflects individual differences in cochlear response times," J. Acoust. Soc. Am. 96, 3476-3491.

Dong, W., and Cooper, N. P. (2006). "An experimental study into the acousto-mechanical effects of invading the cochlea," J. R. Soc., Interface 3, 561-571.

Dong, W., and Olson, E. S. (2008). "Supporting evidence for reverse cochlear traveling waves," J. Acoust. Soc. Am. 123, 222-240.

Eggermont, J., and Don, M. (1980). "Analysis of the click-evoked brainstem potentials in humans using high-pass noise masking. II. Effect of click intensity," J. Acoust. Soc. Am. 68, 1671-1675.

Elberling, C., Parbo, J., Johnsen, N., and Bagi, P. (1985). "Evoked acoustic emission: Clinical application," Acta Oto-Laryngol. 421, 77-85.

Foxe, J. J., and Stapells, D. R. (1993). "Normal infant and adult auditory brain-stem responses to bone-conducted tones," Audiology 32, 95-109.

Goodman, S., Withnell, R., de Boer, E., Lilly, D., and Nuttall, A. (2004).

"Cochlear delays measured with amplitude-modulated tone-burst-evoked OAEs," Hear. Res. 188, 57-69.

Gorga, M., Kaminski, J., Beauchaine, K., and Jesteadt, W. (1988). “Auditory brainstem responses to tone bursts in normally hearing subjects," J Speech Hear. Res. 31, 87-97.

Grandori, F. (1985). "Nonlinear phenomena in click- and tone-burst-evoked otoacoustic emissions from human ears," Audiology 24, 71-80.

Hall, J. W., Bull, J. M., and Cronau, L. H. (1988). "Hypo- and hyperthermia in clinical auditory brain stem response measurement: Two case reports,' Ear Hear. 9, 137-143.

Hatzopoulos, S., Cheng, J., Grzanka, A., and Martini, A. (2000). "Timefrequency analysis of TEOAE recordings from normals and SNHL patients," Audiology 39, 1-12.

Heil, P., and Irvine, D. R. (1997). "First-spike timing of auditory-nerve fibers and comparison with auditory cortex," J. Neurophysiol. 78, 24382454.

Hoth, S., and Weber, F. (2001). "The latency of evoked otoacoustic emissions: Its relation to hearing loss and auditory evoked potentials," Scand. Audiol. 30, 173-183.

Jasper, H. (1958). "The ten-twenty electrode system of the international federation," Electroencephalogr. Clin. Neurophysiol. 10, 371-375.
Jedrzejczak, W., Blinowska, K., and Konopka, W. (2005). "Time-frequency analysis of transiently evoked otoacoustic emissions of subjects exposed to noise," Hear. Res. 205, 249-255.

Jedrzejczak, W., Blinowska, K., Kochanek, K., and Skarzynski, H. (2008). "Synchronized spontaneous otoacoustic emissions analyzed in a timefrequency domain," J. Acoust. Soc. Am. 124, 3720-3729.

Jewett, D. L., and Williston, J. (1971). “Auditory-evoked far fields averaged from scalp of humans," Brain 94, 681-696.

Kalluri, R., and Shera, C. A. (2007). "Near equivalence of human clickevoked and stimulus-frequency otoacoustic emissions," J. Acoust. Soc. Am. 121, 2097-2110.

Kapadia, S., and Lutman, M. E. (2000). "Nonlinear temporal interactions in click-evoked otoacoustic emissions. II. Experimental data," Hear. Res. 146, 101-120.

Keefe, D. (1998). "Double-evoked otoacoustic emissions. I. Measurement theory and nonlinear coherence," J. Acoust. Soc. Am. 103, 3489-3498.

Kemp, D. T. (1978). "Stimulated acoustic emissions from within the human auditory system,” J. Acoust. Soc. Am. 64, 1386-1391.

Kemp, D. T. (2002). "Exploring cochlear status with otoacoustic emissions: The potential for new clinical applications," in Otoacoustic Emissions: Clinical Applications, edited by M. S. Robinette and T. J. Glattike (Thieme, New York), Chap. 1, pp. 1-47.

Kemp, D., Bray, P., Alexander, L., and Brown, A. (1986). "Acoustic emission cochleography-practical aspects," Scand. Audiol. Suppl. 1, 71-95.

Kiang, N. Y. (1975). "Stimulus representation in the discharge patterns of auditory neurons," in The Nervous System. Volume 3: Human Communication and Its Disorders, edited by E. L. Eagles (Raven, New York), pp. 81-96.

Kim, D. O., and Molnar, C. E. (1979). "A population study of cochlear nerve fibers: Comparison of spatial distributions of average-rate and phase-locking measures of responses to single tones," J. Neurophysiol. 42, 16-30.

Konrad-Martin, D., and Keefe, D. H. (2003). "Time-frequency analysis of transient-evoked stimulus-frequency and distortion-product otoacoustic emissions: Testing cochlear model predictions," J. Acoust. Soc. Am. 114, 2021-2043.

Long, G., and Talmadge, C. (1997). "Spontaneous otoacoustic emission frequency is modulated by heartbeat," J. Acoust. Soc. Am. 102, 2831-2848. Lucertini, M., Moleti, A., and Sisto, R. (2002). "On the detection of early cochlear damage by otoacoustic emission analysis," J. Acoust. Soc. Am. 111, 972-978.

Moleti, A., and Sisto, R. (2008). "Comparison between otoacoustic and auditory brainstem response latencies supports slow backward propagation of otoacoustic emissions," J. Acoust. Soc. Am. 123, 1495-1503.

Moleti, A., Sisto, R., and Paglialonga, A. (2008). "Transient evoked otoacoustic emission latency and estimates of cochlear tuning in preterm neonates," J. Acoust. Soc. Am. 124, 2984-2994.

Moleti, A., Sisto, R., Tognoloa, G., Parazzini, M., and Ravazzani, P. (2005). "Otoacoustic emission latency, cochlear tuning, and hearing functionality in neonates," J. Acoust. Soc. Am. 118, 1576-1584.

Møller, A. (1994). "Neural generators of auditory evoked potentials," in Principles and Applications in Auditory Evoked Potentials, edited by J. T. Jacobson (Allyn and Bacon, Massachusetts).

Møller, A. R., and Jannetta, P. J. (1983). "Interpretation of brainstem auditory evoked-potentials: Results from intracranial recordings in humans," Scand. Audiol. 12, 125-133.

Murray, J. G., Cohn, E. S., Harker, L. A., and Gorga, M. P. (1998). "Tone burst auditory brain stem response latency estimates of cochlear travel time in Meniere's disease, cochlear hearing loss, and normal ears," Am. J. Otol. 19, 854-859.

Narayan, S. S. (1991). "Comparison of latencies of N1 and transient evoked otoacoustic emissions: An evaluation of reverse travel in the cochlea," Ph.D. thesis, Purdue University, West Lafayette, IN.

Neely, S., Norton, S., Gorga, M., and Jesteadt, W. (1988). "Latency of auditory brain-stem responses and otoacoustic emissions using tone-burst stimuli," J. Acoust. Soc. Am. 83, 652-656.

Norton, S., and Neely, S. (1987). "Tone-burst-evoked otoacoustic emissions from normal-hearing subjects," J. Acoust. Soc. Am. 81, 1860-1872.

Probst, R., Coats, A., Martin, G., and Lonsbury-Martin, B. (1986). "Spontaneous, click-, and toneburst-evoked otoacoustic emissions from normal ears," Hear. Res. 21, 261-275.

Puria, S. (2003). "Measurements of human middle ear forward and reverse acoustics: Implications for otoacoustic emissions," J. Acoust. Soc. Am. 113, 2773-2789. 
Recio, A., Rich, N. C., Narayan, S. S., and Ruggero, M. A. (1998). "Basilarmembrane responses to clicks at the base of the chinchilla cochlea," J Acoust. Soc. Am. 103, 1972-1989.

Ren, T. (2004). "Reverse propagation of sound in the gerbil cochlea," Nat. Neurosci. 7, 333-334.

Ren, T., and Nuttall, A. L. (2006). "Cochlear compression wave: an implication of the Allen-Fahey experiment," J. Acoust. Soc. Am. 119, 1940 1942.

Riedel, H., Granzow, M., and Kollmeier, B. (2001). "Single-sweep-based methods to improve the quality of auditory brain stem responses Part II: Averaging methods," Z. Fuer Audiologie, Audiological Acoust. 40, 62-85.

Ruggero, M. A., and Temchin, A. N. (2007). "Similarity of traveling-wave delays in the hearing organs of humans and other tetrapods," J. Assoc. Res. Otolaryngol. 8, 153-166.

Ruggero, M. A., Rich, N. C., Recio, A., Narayan, S. S., and Robles, L. (1997). "Basilar-membrane responses to tones at the base of the chinchilla cochlea," J. Acoust. Soc. Am. 101, 2151-2163.

Schönweiler, R., Neumann, A., and Ptok, M. (2005). "Frequency specific auditory evoked responses. Experiments on stimulus polarity, sweep frequency, stimulus duration, notched-noise masking level, and threshold estimation in volunteers with normal hearing," HNO 53, 983-994.

Şerbetçioğlu, M. B., and Parker, D. J. (1999). "Measures of cochlear travelling wave delay in humans: I. Comparison of three techniques in subjects with normal hearing," Acta Oto-Laryngol. 119, 537-543.

Shera, C. A., and Guinan, J. J. (1999). "Evoked otoacoustic emissions arise by two fundamentally different mechanisms: A taxonomy for mammalian OAEs," J. Acoust. Soc. Am. 105, 782-798.

Shera, C. A., and Guinan, J. J. (2003). "Stimulus-frequency-emission group delay: A test of coherent reflection filtering and a window on cochlear tuning," J. Acoust. Soc. Am. 113, 2762-2772.

Shera, C. A., Tubis, A., and Talmadge, C. L. (2005). "Coherent reflection in a two-dimensional cochlea: Short-wave versus long-wave scattering in the generation of reflection-source otoacoustic emissions," J. Acoust. Soc. Am. 118, 287-313.

Shera, C. A., Tubis, A., and Talmadge, C. L. (2008). "Testing coherent reflection in chinchilla: Auditory-nerve responses predict stimulusfrequency emissions," J. Acoust. Soc. Am. 124, 381-395.

Shera, C. A., Tubis, A., Talmadge, C. L., de Boer, E., Fahey, P. F., and Guinan, J. J. (2007). "Allen-Fahey and related experiments support the predominance of cochlear slow-wave otoacoustic emissions," J. Acoust. Soc. Am. 121, 1564-1575.

Siegel, J. H., Cerka, A. J., Recio-Spinoso, A., Temchin, A. N., Van Dijk, P., and Ruggero, M. A. (2005). "Delays of stimulus-frequency otoacoustic emissions and cochlear vibrations contradict the theory of coherent reflection filtering," J. Acoust. Soc. Am. 118, 2434-2443.

Sisto, R., and Moleti, A. (2007). "Transient-evoked otoacoustic emission latency and cochlear tuning at different stimulus levels," J. Acoust. Soc. Am. 122, 2183-2190.

Sisto, R., and Moleti, A. (2002). "On the frequency dependence of the otoacoustic emission latency in hypoacoustic and normal ears," J. Acoust. Soc. Am. 111, 297-308.

Sisto, R., Moleti, A., and Shera, C. A. (2007). "Cochlear reflectivity in transmission-line models and otoacoustic emission characteristic time delays," J. Acoust. Soc. Am. 122, 3554-3561.

Stapells, D. R. (1994). "Low-frequency hearing and the auditory brainstem response," Am. J. Audiol. 7, 11-13.

Stapells, D. R., and Picton, T. W. (1981). "Technical aspects of brainstem evoked potential audiometry using tones," Ear Hear. 2, 20-29.

Stover, L., and Norton, S. (1993). "The effects of aging on otoacoustic emissions," J. Acoust. Soc. Am. 94, 2670-2681.

Stürzebecher, E., Cebulla, M., Elberling, C., and Berger, T. (2006). "New efficient stimuli for evoking frequency-specific auditory steady-state responses," J. Am. Acad. Audiol 17, 448-461.

Suzuki, T., and Horiuchi, K. (1981). "Rise time of pure-tone stimuli in brain stem response audiometry," Audiology 20, 101-112.

Thornton, A., Lineton, B., Baker, V., and Slaven, A. (2006). "Nonlinear properties of otoacoustic emissions in normal and impaired hearing," Hear. Res. 219, 56-65.

Tognola, G., Grandori, F., and Ravazzani, P. (1997). "Time-frequency distributions of click-evoked otoacoustic emissions," Hear. Res. 106, 112122.

von Békésy, G. (1960). Experiments in Hearing (McGraw Hill, New York). Wilson, J. P. (1980a). "Evidence for a cochlear origin for acoustic reemission, threshold fine structure and tonal tinnitus," Hear. Res. 2, 233252 .

Wilson, J. P. (1980b). "Model for cochlear echoes and tinnitus based on an observed electrical correlate," Hear. Res. 2, 527-532.

Wit, H., and Ritsma, R. (1980). "Evoked acoustical responses from the human ear: Some experimental results," Hear. Res. 2, 253-261.

Wit, H., van Dijk, P., and Avan, P. (1994). "Wavelet analysis of real ear and synthesized click evoked otoacoustic emissions," Hear. Res. 73, 141-147.

Zweig, G., and Shera, C. (1995). "The origin of periodicity in the spectrum of evoked otoacoustic emissions," J. Acoust. Soc. Am. 98, 2018-2047. 